\title{
Viscoelastic behavior of cardiomyocytes carrying LMNA mutations
}

\author{
Daniele Borin ${ }^{\mathrm{a}}$, Brisa Peña ${ }^{\mathrm{b}}$, Matthew R.G. Taylor ${ }^{\mathrm{b}}$, Luisa Mestroni ${ }^{\mathrm{b}}$, Romano Lapasin ${ }^{\mathrm{a}}$ and \\ Orfeo Sbaizero ${ }^{\text {a,* }}$ \\ ${ }^{a}$ Department of Engineering and Architecture, University of Trieste, Trieste, Italy \\ ${ }^{\mathrm{b}}$ Cardiovascular Institute, University of Colorado Anschutz Medical Campus - Aurora, Co., USA
}

Received 17 September 2019

Accepted 6 February 2020

\begin{abstract}
.
BACKGROUND: Laminopathies are genetic diseases caused by mutations in the nuclear lamina.

OBJECTIVE: Given the clinical impact of laminopathies, understanding mechanical properties of cells bearing lamin mutations will lead to advancement in the treatment of heart failure.

METHODS: Atomic force microscopy (AFM) was used to analyze the viscoelastic behavior of neonatal rat ventricular myocyte cells expressing three human lamin A/C gene (LMNA) mutations.

RESULTS: Cell storage modulus was characterized, by two plateaus, one in the low frequency range, a second one at higher frequencies. The loss modulus instead showed a "bell" shape with a relaxation toward fluid properties at lower frequencies. Mutations shifted the relaxation to higher frequencies, rendering the networks more solid-like. This increase of stiff ness with mutations (solid like behavior) was at frequencies around $1 \mathrm{~Hz}$, close to the human heart rate.

CONCLUSIONS: These features resulted from a combination of the properties of cytoskeleton filaments and their temporary cross-linker. Our results substantiate that cross-linked filaments contribute, for the most part, to the mechanical strength of the cytoskeleton of the cell studied and the relaxation time is determined by the dissociation dynamics of the cross-linking proteins. The severity of biomechanical defects due to these LMNA mutations correlated with the severity of the clinical phenotype.
\end{abstract}

Keywords: Viscoelasticity, lamin A/C, AFM, cardiomyocytes, storage modulus, loss modulus

\section{Introduction}

A living cell is one of the most evolved materials. The mechanical integrity of healthy cells is meticulously regulated to ensure that cells are solid enough to maintain tissue shape but also being fluid enough to allow dynamic remodeling. Although it is obvious that changing to a complete fluid behavior would be disastrous, yet cells need to dynamically regulate their viscoelastic properties to withstand physiological forces while still allowing motion, growth, and remodeling. This change in the cell

\footnotetext{
* Corresponding author: Orfeo Sbaizero, PhD., Department of Engineering and Architecture, University of Trieste, Via Valerio 10, 34127 Trieste, Italy. Tel.: (+39) 040 5583770; Fax: (+39) 040572044; E-mail: sbaizero@ units.it.
} 
viscoelastic behavior is achieved by a dynamic and continuous reorganization of the cytoskeleton, which is the cell putative load bearing structure [1]. The cytoskeleton elements (actin filaments, microtubules, intermediate filaments) have diff erent mechanical properties and distributions throughout the cell, with each one playing a distinctive part in the cell's mechanics [2,3]. Although there are the tools to identify these filaments in the cytoskeletal networks and study their biophysical and mechanical properties, still there is a deficiency in understanding the mechanical properties of the whole assembly. This knowledge is therefore essential to understand the interconnections between biochemical alterations in cells and the resulting mechanical properties. Furthermore, cells show a number of remarkable dynamic properties in response to external stresses. Exposed to swift deformation, the cytoskeleton develops high levels of stress, while it can easily deform under low strain rates with rather little increase in stress [4]. Moreover, at fixed deformation (or load), a stress relaxation (or creep) behavior is observed [4,5]. Previous studies showed that the actin filaments, cross-linked by actin binding proteins, behave as an entangled network of a semiflexible biopolymer [6] and the kinetics of these physical cross-links is thought to be primarily responsible for tuning the viscoelastic properties of the actin network [7]. While the cross-links stiff en the actin network, their transient nature [8-10] introduces time scale dependent viscoelastic properties [11- 13]. Thus, results differ depending on the probed time seale and can be better quantified measuring the frequency-dependent mechanical response or viscoelasticity of the network [14].

Since it has been shown that "pathological" cells are different from healthy cells in many aspects, including cell growth, adhesion, morphology and organization of the cytoskeleton structure [15-17], the relevance of cell biophysical/biomechanics studies (elastic and viscous components of the rheological response of the cell) is crucial for understanding human disease and improvement of drugs [18]. If the whole-cell mechanical properties have to be assessed, the nucleus as well as the cytoskeleton must be considered since the two entities are not unconnected but linked by the "linkers of the nucleoskeleton and cytoskeleton" (LINC) [19]. The LINC complex connects the nuclear lamina to the cytoskeleton providing at the same time, nuclear anchorage, mechanical stress sensing and resistance to pathologic deformation of the cell [19-22]. In particular, in the nucleus the most important load bearing proteins are the lamins A/C (encoded by LMNA genes), which form a scaff old under the inner nuclear membrane, providing mechanical rigidity to the nucleus and connecting it structurally to the cytoskeleton through the LINC. More than 400 diff erent LMNA mutations causing at least 11 human diseases, termed as laminopathies have being discovered [23]. In this work, we have examined the viscoelastic properties of cardiomyocytes carrying three different LMNA mutations: Glu161Lys (E161K, rs28933093), Asn195Lys (N195K, rs28933091) and Asp192Gly (D192G, rs57045855) compared to control cardiomyocytes (non infected, NI) and cardiomyocytes expressing the human wild type lamin (WT). Diseases due to these mutations lead to phenotypes with blebs in the nuclear envelope, abnormally shaped nuclei, increased nuclear stiff ness, reduced adhesion [24-26].

\section{Materials and methods}

\subsection{Isolation and culture of ventricular cardiomyocytes from neonatal rat(NRVMS)}

All animal studies were performed according to the guidelines of the University of Colorado Denver Animal Care and Use Committee. All animal experiments were done using all achievable methods to ease or minimize possible pain, suff ering or distress, and improve animal welfare. Animals have been provided with housing in an environment, with at least some freedom of movement, food, water and daily care 


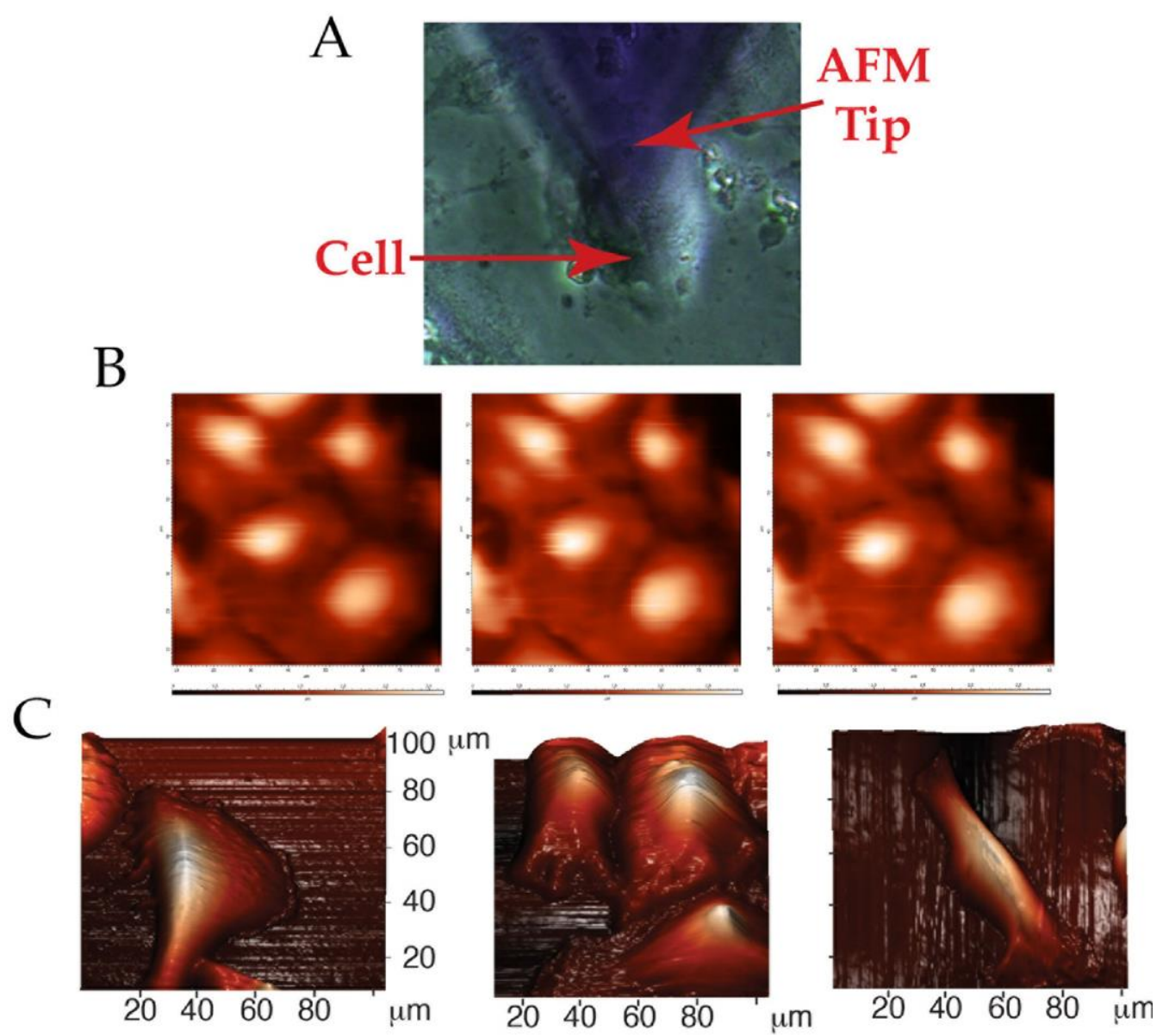

Fig. 1. AFM cells images. (A) AFM tip on top of a cell just before the test start, (B) the 2D AFM cells images and, (C) the corresponding $3 \mathrm{D}$ cell reconstruction.

and cleaning. The well-being and state of health of the animals were controlled by experienced persons, dedicated to the management of the Animal House. Experiments have been performed exclusively by skilled authorized persons.

Primary cultures of neonatal rat ventricular cardiomyocytes (NRVMs) were isolated and cultured from 1-3 days old Sprague Dawley rat pups (Charles River), following decapitation by enzymatic digestion as previously described with minor modifications [27-30]. Briefly, ventricles were separated from the atria using scissors and then dissociated in CBFHH (calcium and bicarbonate-free Hanks with Hepes) buff er containing $0.5 \mathrm{mg} / \mathrm{ml}$ of Collagenase type 2 (Worthington, Biochemical Corporation), and 1 $\mathrm{mg} / \mathrm{ml}$ of Pancreatine (Sigma). Cardiomyocytes were enriched ( $>90 \%$ purity) over non-myocytes by a pre-plating step on 100-mm dishes in Minimum Essential Media (MEM), supplemented with 5\% bovine calf serum and $2 \mathrm{mg} / \mathrm{ml}$ vitamin B12 (Sigma). Myocytes that were either in solution or lightly attached were then separated from the adherent stromal cells by gentle mechanical disaggregation and subsequently plated at a density of $2 \times 10^{5}$ cells/ml in primary petri dishes (Falcon) or in multichambered slides coated with 
A.
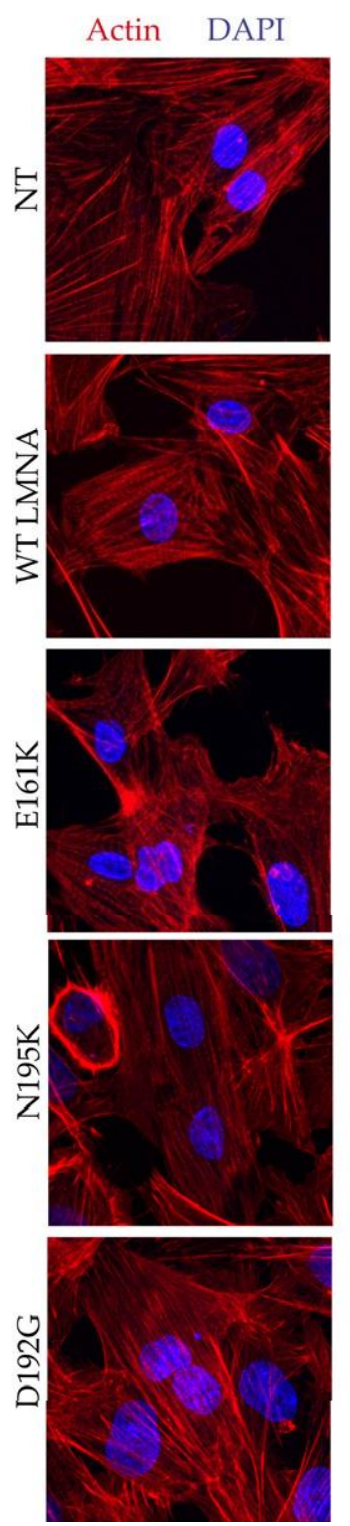

B.

Actin DAPI
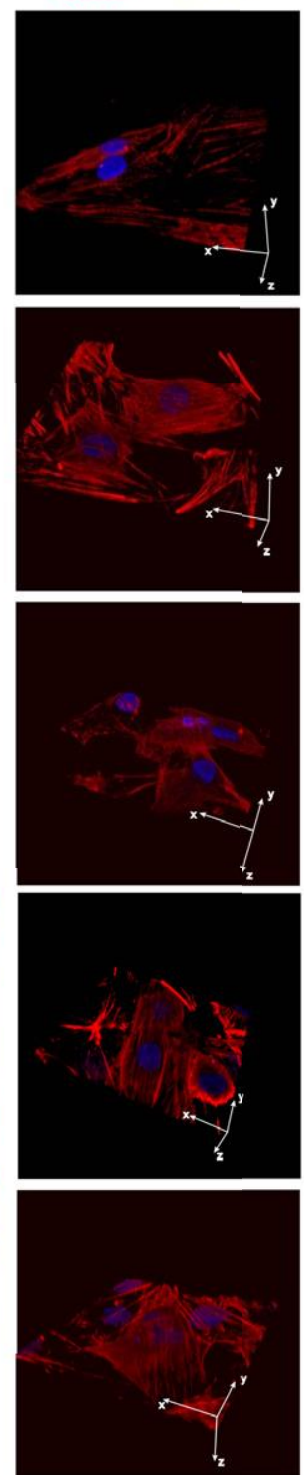
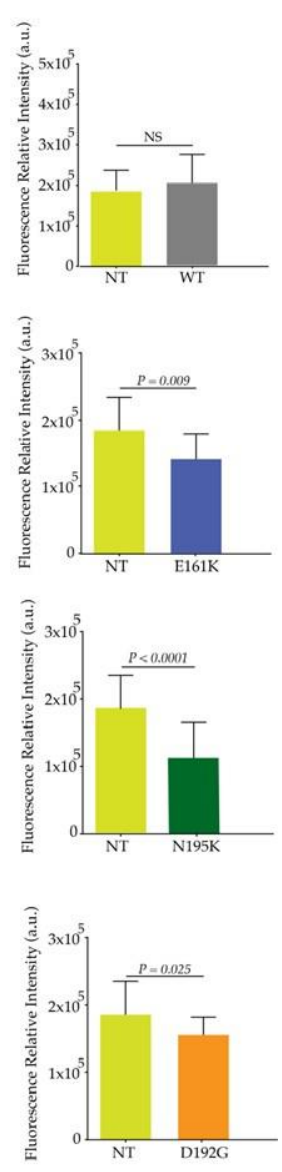

Fig. 2. Actin network in NRVMs. (A) Confocal immunofluorescence microscopy representative images of NVRMs and mutants (E161K, D192G, N195K). Cells stained for actin-stress fibers with phalloidin, (which specifically stains F-actin, red) and the nuclei stained with DAPI (blue). (B) 3D reconstruction of a Z-stack acquired in a range of $9.81 \mu \mathrm{m}$. Cardiomyocytes expressing mutant Lamin protein show an alteration of both density and thickness of cytoskeletal actin microfilaments compared to not infected (NI) or infected with wild-type (WT) LMNA. (C) Quantification of red fluorescence relative intensity $(n=3$ optical fields for each experimental condition), and appearance of labeled actin filaments length. These data confirm a reduction of this cytoskeleton component in mutated NRVM compared to both NI and WT cells. (NI $n=38$ fibers; WT $n=40$ fibers; Mutated $n$ $=67$ fibers) and thickness (NI $n=67$ fibers; WT $n=86$ fibers; Mutated $n=82$ fibers). All images were acquired under identical conditions (independent experiments, $n=4$ ). 
$0.1 \%$ gelatin (Sigma) and cultured as previously described [29,30]. After $18 \mathrm{~h}$, the culture medium was changed and cells were subjected to infection with the relevant adenoviral-LMNA constructs.

\subsection{Isolation Adenoviral constructs and infection}

Methods for adenoviral infection have been previously reported [24,25]. In brief, shuttle constructs were generated in Dual CCM plasmid DNA containing GFP gene and human LMNA cDNA. Constructs were bicistronic with the two inserts (LMNA and GFP) driven by two diff erent CMV promoters to identify cells expressing LMNA protein using GFP as a marker of cellular infection. NRVMs were infected by adenoviruses at 50 multiplicity of infection (MOI) in serum free medium; 6 hours post-infection, complete medium was replaced to cardiomyocytes and the cells were incubated at $37{ }^{\circ} \mathrm{C}$ and $5 \% \mathrm{CO}_{2}$. Previous control experiments have shown that GFP transfection and expression did not aff ect endpoints of interest in NRVM in our experimental conditions [25].

\subsection{Atomic force microscopy determination of cellular biomechanics}

A JPK NanoWizard 4a BioScience AFM was used to acquire the viscoelastic behavior. Since cells can be in diff erent states and hence display dissimilar properties that can be problematic to compare (i) AFM experiments were always completed on the same day after the adenoviral infection, (ii) several measurements from distinctive cells have been gathered to control for unevenness and 'average' data determined, and (iii) cells were examined for morphological details. An optical light microscope was used for cell selection throughout the tests thus cardiomyocytes were selected based on the morphological aspect compared to the possible presence of fibroblasts. The AFM was equipped with PetriDishHeater ${ }^{\mathrm{T}}$ tool to operate in liquid at controlled temperature $\left(37^{\circ} \mathrm{C}\right)$. A cantilever modified with a spherical gold probe (diameter of $\sim 5.5 \mu \mathrm{m}, \mathrm{CP}-\mathrm{PNPL}-\mathrm{Au}-\mathrm{C}$, NanoAndMore $\mathrm{GmbH}$ ) was used to precisely apply a compression force to the nucleus. AFM probes were cleaned, prior to the indentation experiments, by embedding them in Tween ( $2 \%$ for $30 \mathrm{~min}$ ) and then rinsed in milliQ water and ethanol, in order to remove contaminant molecules adsorbed on the probe surface.

For each investigated area, a preliminary scan was made to assess the cell morphology and the nuclear position, which corresponds to the highest portion of the cell. The distal regions, away from the nucleus, were avoided since measurements performed on top of the nucleus are less affected by artifacts due to the substrate stiff ness. In order to avoid cell movement (at low compression speed) or hydrodynamic force contribution (significant at high speed) and possible artefacts due to substrate stiff ness and/or due to hydrodynamic forces, indentations were performed above the nucleus, at the constant speed of 1 $\mu \mathrm{m} / \mathrm{s}$ for approach and withdrawal of the cantilever. Test duration was never longer than $45-50 \mathrm{~min}$ to ensure cell viability.

\subsection{Stress relaxation}

A common method for determining the viscoelastic characteristics of a material is to measure the stress relaxation response to a prescribed displacement. In this AFM-based study, we indented a cell with an approximate step displacement and recorded the resulting force response over time. In this case the stress relaxation tests is called "constant height mode" [31]. The indentation depth for each cell was set to the $30 \%$ of its initial height and the movement of the piezo scanner in the $\mathrm{Z}$ direction was corrected with the deflection of the cantilever during the cell compression. The indentation depth of $30 \%$ was chosen as a 
trade-off between the highly noisy relaxation data at lower indentations and the purpose to stress all the cell components (membrane, cytoskeleton and nucleus) [32]. After indenting the cell, the cantilever base position was kept constant for a fixed time of $60 \mathrm{~s}$ to minimize drift, while the cantilever force was recorded with time. Finally, the AFM tip was retracted until full detachment from the cell. The stress relaxation measurement was performed only one time per cell to avoid potential artifacts due to the prolonged stress status.

Force data relative to the relaxation phase were expressed in terms of stress by dividing them by the contact area (represented by the projection of the spherical cap of the probe in contact with the cell after the indentation, considered constant during the relaxation phase). Stress data were then divided by deformation (indentation/initial cell height) to give a relaxation modulus $E(t)$ that can be compared with models normally used to fit linear viscoelastic behaviors. Conventional viscoelastic models are obtained from the combination of springs (elastic elements) and dashpots (viscous elements) arranged in parallel and/or in series. In this work, the standard linear solid (SLS) model, also called Zener's model, has been chosen to correlate the viscoelastic responses of cells obtained from AFM tests, by virtue of its simplicity. Indeed, the model derives from the parallel arrangement of a spring and a Maxwell element, in its turn made of a spring and a dashpot combined in series. Accordingly, the relaxation modulus data are correlated with the following exponential expression:

$$
\begin{aligned}
& \left(-\frac{\square}{-1}\right)
\end{aligned}
$$

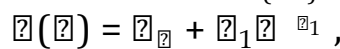

where $E_{e}$ is the equilibrium modulus, deriving from the spring element and corresponding to asymptotic value of $E(t)$ for $t \rightarrow \infty, E_{1}$ is the elastic moduli of the Maxwell element, $]_{1}$ is the corresponding relaxation time.

\subsection{Storage and loss moduli calculation}

In the linear viscoelastic regime, where the Boltzmann superposition is applicable, knowledge of the relaxation modulus makes it possible to predict the rheological responses in any arbitrary deformation history, by resorting to the integral version of the linear generalized viscoelastic model, since the relaxation modulus plays the role of a predictive constitutive model parameter [33]. Consequently, the viscoelastic responses under steady oscillatory motion can be predicted from the relaxation modulus, as long as the deformations imposed in both relaxation test and oscillatory conditions are sufficiently small, within the linear regime. In the case of the Zener model used to fit the relaxation data, the following expressions are derived for the storage $\left(E^{\prime}\right)$ and loss moduli $\left(E^{\prime \prime}\right)$ as a function of the (angular) frequency 0 [34]:

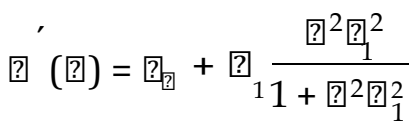

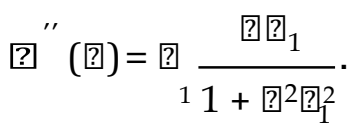

Such equations can help to make explicit the contributions of the elastic and viscous components to the total rheological response starting from the relaxation tests, which are assumed to be performed in quasi linear conditions. The loss tangent, defined as the ratio between the loss modulus and the storage modulus $\left(\tan 0=E^{\prime \prime} E^{\prime}\right)$, can be used as an inverse index of elasticity, the smaller values indicating a more elastic character of the material response. 


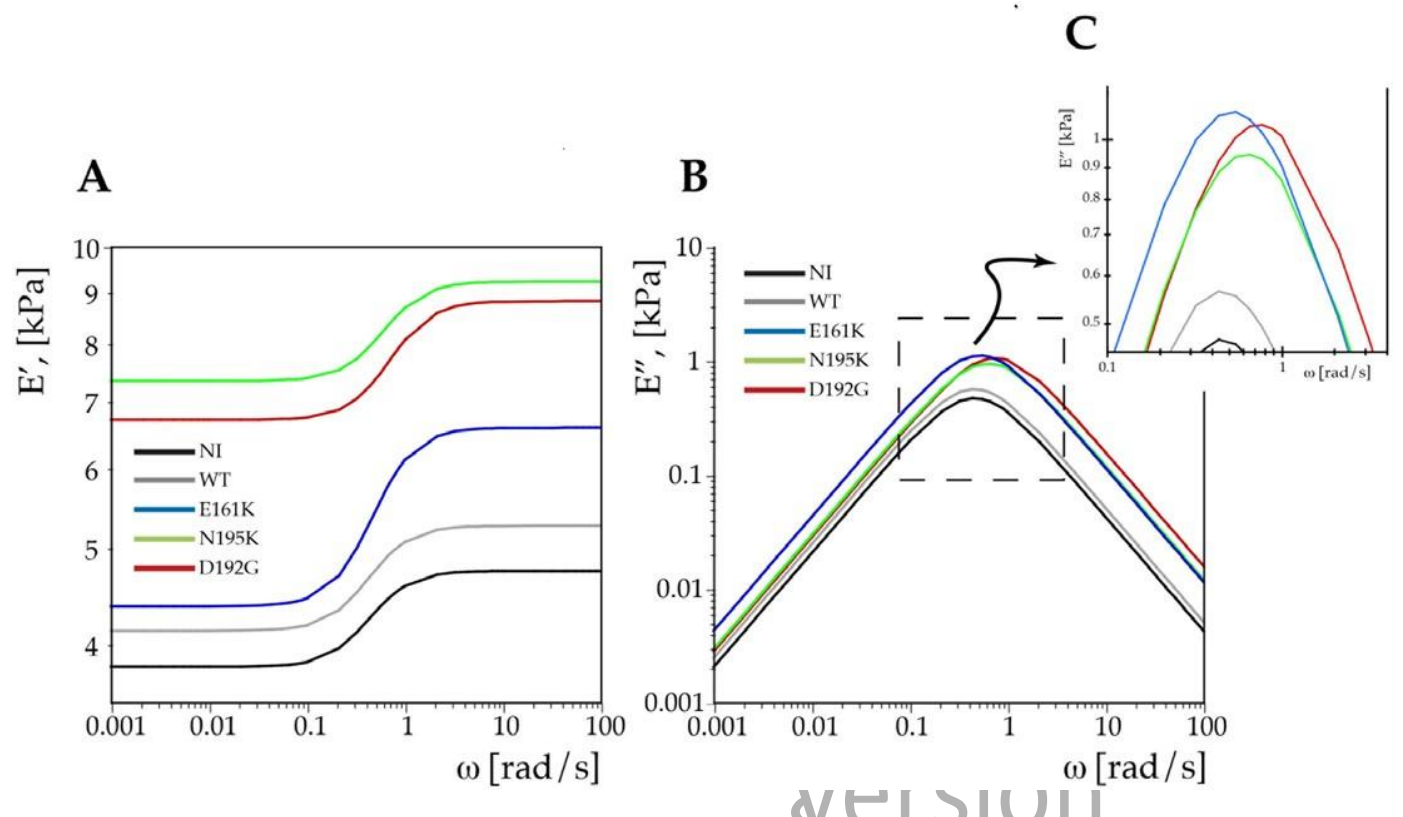

Fig. 3. (A) storage modulus for the cell lines tested, (B) loss modulus, (C) detail of the loss modulus.

\subsection{Statistics}

Several tests from at least 20 cells were collected to obtain 'average' data for each condition of interest. The curves presented in the Figs 3 are the average of the collected family of curves. AFM data were tested with the Shapiro-Wilk normality test $($ ? $=0.05$ ) and their statistical significance, reported through the $p$ value, was probed applying the Kruskal-Wallis test; treated cells were compared to the control using the Dunnett's correction for multiple comparisons. A confidence interval of $95 \%$ (corresponding to ? value of 0.05 ) was used in order to designate differences as statistically significant. Statistical data are reported in Supplemental materials S1.

\section{Results}

The efficiency of LMNA transduction was already determined in previous studies [24,25]. Increases in cell death due to the transduction were not observed during the course of our studies (data not shown). Figure 1 shows (A) an AFM tip on top of a cell just before the test start, (B) the 2D AFM cells images and $(\mathrm{C})$ the corresponding $3 \mathrm{D}$ cell reconstruction. Confocal microscopy images (Fig. 2A-B) showed that in both control and wild type NRVMs, the actin microfilaments appeared to be well organized and regularly distributed. On the contrary, mutants NRVMs display attenuation and disarray of the actin microfilaments. A quantitative measurement of actin filaments thickness (Fig. 2C) showed a substantial reduction of this cytoskeletal component in Mutated NRVMs compared to NI and WT cells. Due to their cross-linked cytoskeletal network, cells exhibit viscoelastic behavior, having the ability to store energy but also to flow when set under small forces. The elastic response can be characterized by the storage modulus $\left(E^{\prime}\right)$, a quantity that assesses the ability to "store" elastic energy that can ultimately be recovered, while 
the loss modulus $\left(E^{\prime \prime}\right)$ measures the amount of energy that is the heat dissipated in local flow conditions occurring inside the cell.

Figure $3 \mathrm{~A}$ and $\mathrm{B}$ show the values of the viscoelasticity moduli, $E^{\prime}$ and $E^{\prime \prime}$, as a function of the angular frequency for the cell lines studied. The curves are the mean values of the $E^{\prime}$ and $E^{\prime \prime}$, respectively. Both the storage and the loss moduli exhibit a pronounced frequency dependence however, at the evaluated frequencies, storage modulus values $\left(E^{\prime}\right)$ were always higher than those of the loss modulus $\left(E^{\prime \prime}\right)$, which indicates that for all cell lines considered, elastic properties prevailing over the viscous ones. Thus, these cells behavior can be classified as a gel [35]. The storage modulus increases with the frequency while the loss modulus goes through a maximum then it decreases as frequency increases. As far as the storage modulus is concerned (Fig. 3A), all samples show an initial linear region where it is independent of the frequency followed by an increase of this modulus in the frequency region in which the loss modulus reaches its maximum (between 0.1 and $3 \mathrm{rad} / \mathrm{s}$ ) and whose position along the frequency axis corresponds to the inverse of the relaxation time ${ }_{1}$. $^{2}$

The storage modulus for the control NI cardiomyocytes is $3.8 \pm 0.23 \mathrm{kPa}$ at low frequency and increases to $4.74 \pm 0.11 \mathrm{kPa}$ at high frequency $(+24.7 \%)$, for WT and the two mutations N195K and D192G the increases are similar: $26.7 \%, 27 \%$ and $30.5 \%$ respectively; while the E161K mutations shows a larger increase $(50.2 \%)$.

Figure 3B shows the frequency dependence of the loss modulus. For all cell lines the loss modulus displays a maximum, being the viscous contribution given by the only Maxwell element. However, while NI and WT show the same behavior with the frequency, the E161K not only shows the highest peak of the all three mutations but also has higher values at low frequency and lower values at high frequency when compared to the N195K and D192G mutations. Furthermore, for the three mutations the maximum in the loss modulus shifts to higher frequencies compared to NI and WT. The shift is higher for D192G followed by N195K and E161K, respectively(Fig. 3C).

\section{Discussion}

The cell is a heterogeneous system, therefore, assessing its mechanical properties is puzzling. In previous works [24-26] we assessed the elasticity (Young's Modulus) of the three mutations currently studied. The Young's modulus in cells expressing mutant LMNA are significantly higher than NI and WT. More precisely, D192G displayed the highest Young Modulus followed by N195K and E161K. Moreover, mutant cells display extensive actin cytoskeletal network alteration. These biomechanical and structural defects agree with clinical phenotypic severity since the most severe outcome is associated with the D192G variant, while the mildest with the E161K. However, a single elastic Young's modulus is not enough to describe the behavior of a complex polymer system such as a cell's cytoskeleton. The cytoskeleton shows both viscous and elastic response to deformation. For this reason, the frequencydependent viscoelastic behavior is another fundamental aspect which must be accounted for in order to describe the whole cell behavior. The viscoelastic characteristics of the cells have been assessed with stress relaxation tests and can be described by classical phenomenological models (the Zener model in our case) with satisfactory approximation. We preferred to off er an additional but equivalent representation of the viscoelastic behavior in the frequency domain, i.e. using reciprocal time as a reference quantity. Indeed, within this artificial frame we can distinguish more explicitly both the elastic and viscous contributions and their relative weight through the profiles of $E^{\prime}$ and $E^{\prime \prime}$ as well as the characteristic times of the Maxwell 
contribution. The time domain data and a comparison of Zener parameters for the diff erent cases can be found in the Supplemental Material S1.

Since the cytoskeleton is connected to the nucleus through the LINC complex, our measured data reflect the combined eff ects of the nucleus and the cytoskeleton. The cytoskeleton helps to stiff en the nucleus to inhibit extreme nuclear deformation. Both the cytoskeleton and nucleus behavior can be represented by a spring and a damper in the form of the Zener model [36]. The cytoskeleton has a high viscosity therefore its time for strain recovery is longer than that of the nucleus. Therefore, the linkage between cytoskeleton and nucleus (through LINC and lamin) slows down the strain recovery of the nucleus itself. Cells use cross-linkers to the cytoskeleton filaments to form an elastic network that can withstand mechanical load. Therefore, entangled filamentous cytoskeleton components and associated crosslinking proteins play an essential role in governing the mechanical properties of cells and they can also play a critical role in diseases. Furthermore, cross-links are not covalent, they can unbind and rebind having a transient behavior ensuring (i) mechanical compliance at long timescales but also (ii) an elastic response at timescales shorter than the cross-linker off-rate.

Normally the cytoskeleton filaments (mainly actin) are modelled as semiflexible polymer, that is their persistence lengths are roughly on the same order as the contour lengths and therefore the network is considered to be stiff enough to prevent coiling. With the lack of cross-linkers, the cytoskeleton would be an entangled network that behaves like a weak viscoelastic solid. However, the addition of crosslinking proteins dramatically strengthens these networks [11,12,37-40]. Furthermore, the response of these networks depends on the timescale on which they are investigated and is best quantified by a measure of the frequency-dependent mechanical response or yiscoelasticity of the network.

In general terms, the greater is the cell compliance, the easier it is to deform it. In this study, when the stress was applied with the AFM tip, there was a sharp and instantaneous increase in strain, followed by a slight increase (relaxation) over time, which can be attributed to some re-arrangement of network structure (cytoskeleton). On removal of the stress, there was also a corresponding reduction in compliance, with an almost complete recovering to the original states, indicating an elastic gel structure.

The density of cross-linked filaments is determined by the cross-linking proteins present, mainly alphaactinin, filamin, and Arp 2/3 [41-43]. These proteins aff ect both the cell static and dynamic properties, because they transitorily bind to actin. Typical binding times are in the range of 0.2-3 s [43] (comparable with the experimental times of intermediate frequency region. $(0.33-5 \mathrm{rad} / \mathrm{s}) \mathrm{in} \mathrm{Fig.} \mathrm{3).} \mathrm{This} \mathrm{lets} \mathrm{cross-}$ linked actin to behave as an elastic gel on time scales shorter than the binding times and a fluid on longer times.

For all cell lines considered, both $E^{\prime}$ and $E^{\prime \prime}$ depend on frequency $(0.001-100 \mathrm{rad} / \mathrm{s})$. From Fig. 3,

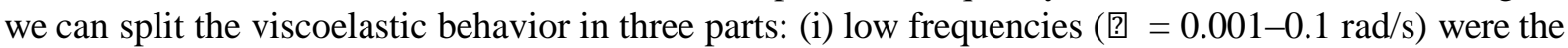
cytoskeleton filaments are displaced relative to each other but the cross links have time to rearrange by dissociating and re-associating and therefore their mechanical eff ects are negligible. In this case entanglements become important and they are the main mechanism of sterically hinder individual filaments. The filaments motion is assumed to be confined inside a tube; reptation dominates the mechanism of relaxation [44]; (ii) high frequencies ( $?=3-100 \mathrm{rad} / \mathrm{s}$ ), where the time scale is very short and the crosslinkers do not have time to unbind, consequently they are put under tension making the whole network more rigid; (iii) the intermediate frequencies ( $0=0.1-3 \mathrm{rad} / \mathrm{s}$ ) where the storage modulus increases and a corresponding maximum of the loss modulus is reached.

The elastic modulus is independent of frequency for $0>3 \mathrm{rad} / \mathrm{s}$ and exhibits relaxation toward a complex, fluid-like behavior at lower frequencies, as shown in Fig. 3A. Furthermore, $E^{\prime}$ shows a transition from a high frequency plateau to a low frequency plateau and a maximum in $E^{\prime \prime}$ that coincides with this 
transition. The high frequency plateau modulus, suggests the combined contributions of both permanent cross-links and transient cross-links. As the oscillation frequency is decreased, the timescale on which the cell is deformed becomes comparable to the characteristic timescale for dissociation of transient crosslinks. At low frequencies or longer times, the transient cross-links are no longer elastically efficient but stress is still endured by the deformation of filaments connected by entanglements. As aforementioned, the loss modulus is characterized by a maximum. The maximum in $E^{\prime \prime}$ which occurs as $E^{\prime}$ transitions between the high and low frequency plateaus (Fig. 3A-B). The relaxation time (frequency) for the transient cross-links, which is determined from the frequency at which the maximum in $E^{\prime \prime}$ occurs, is the same for NI and WT cells but is moved to higher frequencies for the three mutations. A similar eff ect was observed in physical protein hydrogels that also contained entanglements [45]. The position of the maximum in $E^{\prime \prime}$ moves to lower frequencies as the filaments become less able to diff use, either by cross- linking or increasing concentration or length [46] therefore these data confirm that the three mutations are characterized by a smaller actin filaments length as found in previous works [24,26].

We characterized the network linear response by the plateau modulus $E_{0}$, the value of $E^{\prime}$ corresponding to the maximum of the loss modulus. The smallest elastic plateau modulus $E_{0}=4.3 \mathrm{kPa}$ for the NI cells suggests that these cells show the weakest viscoelastic solid of those tested $\left(E_{0}=4.65 \mathrm{kPa}\right.$ for WT; $E_{0}=$ $5.45 \mathrm{kPa}$ for $\mathrm{E} 161 \mathrm{~K} ; E_{0}=8.2 \mathrm{kPa}$ for $\mathrm{N} 195 \mathrm{~K} ; E_{0}=7.8 \mathrm{kPa}$ for D192G) furthermore, mutations heighten the plateau, leading the network properties toward that of a solid. Since storage modulus is much higher than loss modulus, $E^{\prime}$ data at low frequencies provide a quite close estimation of cell Young's modulus. D192G and N195K mutations show the highest Young modulus follow by the E161K and this is in good agreement with the data obtained on the same cells line using the AFM in single cell force spectroscopy mode $[25,26]$.

All the loss moduli exhibit a frequency dependence, with $E^{\prime \prime}$ reaching a maximum before showing a fluid like behavior at lower frequencies. For low frequencies (smaller than the $E^{\prime \prime}$ maximum) all cells relax with the same power: $E^{\prime \prime} \sim$ ? ${ }^{⿴ 囗 十}$ where $0 \approx 1.2-1.4$; a behavior typical of a fluid flow. The frequency of the local maximum in $E^{\prime \prime}$ can be recognized as the relaxation frequency ${ }_{r}$, which characterizes the unbinding rate of cross-linkers [13,47-49]. This can be used to compare the network relaxation associated with the various types of mutations.

The value of ${ }_{r}$ is the same for NI and WT cells ( $\left.{ }_{r} \sim 0.45 \mathrm{rad} / \mathrm{s}\right)$ but it is larger for the mutated cytoskeleton $\left(E 161 \mathrm{~K} 0_{r} \sim 0.55 \mathrm{rad} / \mathrm{s}\right.$; N195K $?_{r} \sim 0.65 \mathrm{rad} / \mathrm{s}$; D192G 目 ${ }_{r} \sim 0.78 \mathrm{rad} / \mathrm{s}$; as shown in Fig. 3C. As aforementioned, the plateau modulus is due to entanglement eff ects that become more marked at longer time scales. If we assume that the cross-linkers dissociation rates follow the Arrhenius equation [48]:

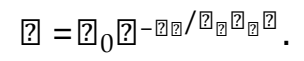

Where, $E_{a}$ is the molar activation energy for the dissociation reaction, $k_{0}$ the rate of unbinding, and $k_{B}$ and $N_{A}$ the Boltzmann and Avogadro constants, respectively. Since the activation energy appears in the exponential, a small diff erence is enough to cause a diff erence in dissociation rate. While mutant cells depict an increased unbinding frequency, NI and WT cells show smaller unbinding frequencies, suggesting an extended timescale for cross-linker unbinding. These results are in good agreement with those shown by Lieleg et al. [11]. In their paper they show that the cross-link kinetics changes the position of both the maximum of the viscous dissipation and the frequency at which the network elasticity $(E)$ starts to drop. Our data show that in mutated cells cross-linkers keep filaments together for shorter times comparted to control cells. This suggests that stress relaxation in the cytoskeleton of mutated cells will occur more 
rapidly, reducing the ability for these cells to resist larger forces than normal. In other words, the reduced binding time shifts the cytoskeleton to a more fluid-like regime.

At low frequencies (smaller than the maximum in $E^{\prime \prime}$ ), the filaments entanglements become more important. An indicator of entanglement is the entanglement length $\left(l_{e}\right)$. For low frequencies, steric and hindrance leads to a frequency-independent elastic plateau modulus over a wide frequency range [50]:

$$
\text { 回 } 0 \text { 回回/回回回 }
$$

where $k_{B}$ and $\mathrm{T}$ are the Boltzmann constant and the absolute temperature, respectively, ? is the mesh size. In our case, for ( $2<0.1 \mathrm{rad} / \mathrm{s}$ ) the three mutations show always a higher frequency-independent elastic plateau modulus meaning that either the mesh size or the entanglement length is smaller in these cells. However, the E161K mutation shows the smallest increase in the low frequency plateau(Fig. 3A).

The contributions of microtubules and intermediate filaments to cell mechanics should also be considered. Intermediate filaments are quasi permanently cross-linked, and the network of microtubules remains unchanged on time scales longer than tens of seconds, thus we can rule out these two cytoskeletal elements as the origin of the observed high frequency dynamic behavior. Still, these two cytoskeletal elements may contribute to the elastic response for longer deformations time ( $>10 \mathrm{~s}=0.62 \mathrm{rad} / \mathrm{s}$, therefore for frequencies lower than the maximum in $\left.E^{\prime \prime}\right)$. It has been shown that the different filament types influence each other by virtue of their presence through their respective viscoelastic responses, for example the presence of surrounding actin reduces the length scale for transverse fluctuations of microtubules [51]. It is therefore interesting to ask whether the presence of MTs modulates actin filament fluctuations.

\section{Conclusions}

Our AFM data show that the viscoelasticity of control and mutant neonatal rat ventricular cardiomyocytes is characterized as far as the storage modulus is concerned, by two elastic plateaus, one in the low frequency range between $0.001 \mathrm{rad} / \mathrm{s}$ and $0.1 \mathrm{rad} / \mathrm{s}$, a second one at higher frequencies $>3 \mathrm{rad} / \mathrm{s}$. All three mutations exhibit some degree of stiff ening (higher values of $E^{\prime}$ ) albeit smallest for the E161K.

The loss modulus instead displays a "bell" shape and a relaxation, at lower frequencies, toward fluid properties. This outcome is due to a combination of the cytoskeleton filaments properties and their transient cross-linker. The plateau at high frequencies is a direct consequence of cross-linking. For the three mutations the plateau was higher however, for the E161K mutation the value was the closest to that of the controls. The frequency of the local maximum in the loss moduli can be recognized as the relaxation frequency, which characterizes the unbinding rate of cross-linkers. We found that unbinding frequency is shifted to shorter time scales compared to control cells, suggesting that mutant cell have a shorter timescale for cross-linker unbinding.

The mutations not only shift the relaxation to higher frequencies, but also generate a pronounced elastic plateau, thus shaping the networks to a more solid-like behavior. It is interesting that these drastic increase of their stiff ness (solid like behavior) is at frequencies around $1 \mathrm{~Hz}$, close to the human heart rate.

Our results substantiate that cross-linked filaments contribute for the most part of the mechanical strength of the cytoskeleton cell studied and the relaxation time is determined by the dissociation dynamics of the cross-linking proteins. 


\section{Author Contributions}

The work presented here was carried out in collaboration between all authors.

Matthew R.G. Taylor, Luisa Mestroni and Orfeo Sbaizero developed the original idea; Orfeo Sbaizero designed methods and experiments and defined the research theme. Matthew R.G. Taylor and Luisa Mestroni provided cell lines. Daniele Borin carried out AFM tests. Daniele Borin and Romano Lapasin analysed the data. Orfeo Sbaizero and Romano Lapasin drafted the manuscript and all authors carried out a critical revision of the manuscript for important intellectual content.

\section{Competing interests}

All authors confirm that there have been no financial or other interests related to the submitted work that (1) could aff ect or have the perception of aff ecting the author's objectivity, or (2) could influence or have the perception of influencing the content of the article.

\section{Funding}

This study was supported by the following grant: Fondation Leducq Transatlantic Network of Excellence 14-CVD 03.

\section{References}

[1] Fletcher DA, Mullins RD. Cell mechanics and the cytoskeleton. Nature. 2010;463(7280):485-492.

[2] Tang DD, Gerlach BD. The roles and regulation of the actin cytoskeleton, intermediate filaments and microtubules in smooth muscle cell migration. Respir Res. 2017;18(1):54.

[3] Pegoraro AF, Janmey P, Weitz DA. Mechanical properties of the cytoskeleton and cells. Cold Spring Harb Perspect Biol. 2017;9(11).

[4] Trepat X, Deng L, An SS, Navajas D, Tschumperlin DJ, Gerthoff er WT et al. Universal physical responses to stretch in the living cell. Nature. 2007;447(7144):592-595.

[5] Matthews BD, Overby DR, Mannix R, Ingber DE. Cellular adaptation to mechanical stress: Role of integrins, Rho, cytoskeletal tension and mechanosensitive ion channels. J Cell Sci. 2006;119(Pt3):508-518.

[6] Jensen MH, Morris EJ, Goldman RD, Weitz DA. Emergent properties of composite semiflexible biopolymer networks. Bioarchitecture. 2014;4(4-5):138-143.

[7] Gardel ML, Nakamura F, Hartwig J, Crocker JC, Stossel TP, Weitz DA. Stress-dependent elasticity of composite actin networks as a model for cell behavior. Phys Rev Lett. 2006;96(8):088102.

[8] Fritzsche M, Erlenkämper C, Moeendarbary E, Charras G, Kruse K. Actin kinetics shapes cortical network structure and mechanics. Sci Adv. 2016;2(4):e1501337.

[9] Fritzsche M, Lewalle A, Duke T, Kruse K, Charras G. Analysis of turnover dynamics of the submembranous actin cortex. Mol Biol Cell. 2013;24(6):757-767.

[10] Ferrer JM, Lee H, Chen J, Pelz B, Nakamura F, Kamm RD et al. Measuring molecular rupture forces between single actin filaments and actin-binding proteins. Proc Natl Acad Sci USA. 2008;105(27):9221-9226.

[11] Lieleg O, Claessens MM, Luan Y, Bausch AR. Transient binding and dissipation in cross-linked actin networks. Phys Rev Lett. 2008;101(10):108101.

[12] Lieleg O, Schmoller KM, Claessens MM, Bausch AR. Cytoskeletal polymer networks: Viscoelastic properties are determined by the microscopic interaction potential of cross-links. Biophys J. 2009;96(11):4725-4732.

[13] Broedersz CP, Depken M, Yao NY, Pollak MR, Weitz DA. MacKintosh FC. Cross-link-governed dynamics of biopolymer networks. Phys Rev Lett. 2010;105(23):238101. 
[14] Fabry B, Maksym GN, Butler JP, Glogauer M, Navajas D, Fredberg JJ. Scaling the microrheology of living cells. Phys Rev Lett. 2001;87(14):148102.

[15] Ketene AN, Schmelz EM, Roberts PC, Agah M. The eff ects of cancer progression on the viscoelasticity of ovarian cell cytoskeleton structures. Nanomedicine. 2012;8(1):93-102.

[16] Darling EM, Zauscher S, Guilak F. Viscoelastic properties of zonal articular chondrocytes measured by atomic force microscopy. Osteoarthritis Cartilage. 2006;14(6):571-579.

[17] Banerjee A, Rathee V, Krishnaswamy R, Bhattacharjee P, Ray P, Sood AK et al. Viscoelastic behavior of human lamin A proteins in the context of dilated cardiomyopathy. PLoS One. 2013;8(12):e83410.

[18] Suresh S. Biomechanics and biophysics of cancer cells. Acta Biomater. 2007;3(4):413-438.

[19] Stroud MJ, Banerjee I, Veevers J, Chen J. Linker of nucleoskeleton and cytoskeleton complex proteins in cardiac structure, function, and disease. Circ Res. 2014;114(3):538-548.

[20] Cattin ME, Muchir A, Bonne G. 'State-of-the-heart' of cardiac laminopathies. Curr Opin Cardiol. 2013;28(3):297-304.

[21] Schreiber KH, Kennedy BK. When lamins go bad: Nuclear structure and disease. Cell. 2013;152(6):1365-1375.

[22] Lammerding J, Wolf K. Nuclear envelope rupture: Actin fibers are putting the squeeze on the nucleus. J Cell Biol. 2016;215(1):5-8.

[23] Shimi T, Butin-Israeli V, Adam SA, Goldman RD. Nuclear lamins in cell regulation and disease. Cold Spring Harb Symp Quant Biol. 2010;75:525-531.

[24] Lanzicher T, Martinelli V, Long CS, Del Favero G, Puzzi L, Borelli M et al. AFM single-cell force spectroscopy links altered nuclear and cytoskeletal mechanics to defective cell adhesion in cardiac myocytes with a nuclear lamin mutation. Nucleus. 2015;6(5):394-407.

[25] Lanzicher T, Martinelli V, Puzzi L, Del Favero G, Codan B, Long CS et al. The cardiomyopathy lamin A/C D192G mutation disrupts whole-cell biomechanics in cardiomyocytes as measured by atomic force microscopy loading-unloading curve analysis. Sci Rep. 2015;5:13388.

[26] Laurini E, Martinelli V, Lanzicher T, Puzzi L, Borin D, Chen SN et al. Biomechanical defects and rescue of cardiomyocytes expressing pathologic nuclear lamins. Cardiovasc Res. 2018;114(6):846-857.

[27] Long CS, Kariya K, Karns L, Simpson PC. Sympathetic modulation of the cardiac myocyte phenotype: Studies with a cell-culture model of myocardial hypertrophy. Basic Res Cardiol. 1992;87(Suppl 2):19-31.

[28] Deng XF, Rokosh DG, Simpson PC. Autonomous and growth factor-induced hypertrophy in cultured neonatal mouse cardiac myocytes. Comparison with rat. Circ Res. 2000;87(9):781-788.

[29] Martinelli V, Cellot G, Toma FM, Long CS, Caldwell JH, Zentilin L et al. Carbon nanotubes promote growth and spontaneous electrical activity in cultured cardiao myocytes. Nano Lett. 2012;12(4):1831-1838.

[30] Martinelli V, Cellot G, Fabbro A, Bosi S, Mestroni L, Ballerini L. Improving cardiac myocytes performance by carbon nanotubes platforms. Front Physiol. 2013;4:239.

[31] Moreno-Flores S, Benitez R, VivancoM, Toca-Herrera JL. Stress relaxation and creep on living cells with the atomic force microscope: A means to calculate elastic moduli and viscosities of cell components. Nanotechnology. 2010;21(44):445101.

[32] Borin D, Puzzi L, Martinelli V, Cibinel M, Lapasin R, Sbaizero O. An engineering insight into the relationship of selective cytoskeletal impairment and biomechanics of HeLa cells. Micron. 2017;102:88-96.

[33] Bird RB Dynamics of Polymeric Liquids. In: Byron Bird R et al. (ed)2nd ed. New York, Chichester: Wiley; 1987.

[34] Schmitt C, Hadj Henni A, Cloutier G. Characterization of blood clot viscoelasticity by dynamic ultrasound elastography and modeling of the rheological behavior. J Biomech. 2011;44(4):622-629.

[35] Rao MA. Rheology of Fluid and Semisolid Foods: Principles and Applications. Gaithersburg, MD: Aspen Publishers; 1999.

[36] Wang X, Liu H, Zhu M, Cao C, Xu Z, Tsatskis Y et al. Mechanical stability of the cell nucleus - roles played by the cytoskeleton in nuclear deformation and strain recovery. J Cell Sci. 2018;131(13).

[37] Xu J, Tseng Y, Wirtz D. Strain hardening of actin filament networks. Regulation by the dynamic cross-linking protein alpha-actinin. J Biol Chem. 2000;275(46):35886-35892.

[38] Esue O, Tseng Y, Wirtz D. Alpha-actinin and filamin cooperatively enhance the stiff ness of actin filament networks. PLoS One. 2009;4(2):e4411.

[39] Schmoller KM, Lieleg O, Bausch AR. Structural and viscoelastic properties of actin/filamin networks: Cross-linked versus bundled networks. Biophys J. 2009;97(1):83-89.

[40] Kasza KE, Broedersz CP, Koenderink GH, Lin YC, Messner W, Millman EA et al. Actin filament length tunes elasticity of flexibly cross-linked actin networks. Biophys J. 2010;99(4):1091-1100.

[41] Eichinger L, Köppel B, Noegel AA, Schleicher M, Schliwa M, Weijer K et al. Mechanical perturbation elicits a phenotypic diff erence between Dictyostelium wild-type cells and cytoskeletal mutants. Biophys J. 1996;70(2):1054-1060. 
[42] Mullins RD, Heuser JA, Pollard TD. The interaction of Arp2/3 complex with actin: Nucleation, high affinity pointed end capping, and formation of branching networks of filaments. Proc Natl Acad Sci USA. 1998;95(11):6181-6186.

[43] Wottawah F, Schinkinger S, Lincoln B, Ananthakrishnan R, Romeyke M, Guck J et al. Optical rheology of biological cells. Phys Rev Lett. 2005;94(9):098103.

[44] Cates ME. Reptation of living polymers: Dynamics of entangled polymers in the presence of reversible chain-scission reactions. Macromolecules. 1987;2289-2296.

[45] Tang S, Glassman MJ, Li S, Socrate S, Olsen BD. Oxidatively responsive chain extension to entangle engineered protein hydrogels. Macromolecules. 2014;47(2):791-799.

[46] Doi M, Edwards SS. The Theory of Polymer Dynamics. Clarendon; 1986.

[47] Lieleg O, Claessens MM, Heussinger C, Frey E, Bausch AR. Mechanics of bundled semiflexible polymer networks. Phys Rev Lett. 2007;99(8):088102.

[48] Ward SM, Weins A, Pollak MR, Weitz DA. Dynamic viscoelasticity of actin cross-linked with wild-type and diseasecausing mutant alpha-actinin-4. Biophys J. 2008;95(10):4915-4923.

[49] Yao NY, Becker DJ, Broedersz CP, Depken M, Mackintosh FC, Pollak MR et al. Nonlinear viscoelasticity of actin transiently cross-linked with mutant 0 -actinin-4. J Mol Biol.2011;411(5):1062-1071.

[50] Liu J, Gardel ML, Kroy K, Frey E, Hoff man BD, Crocker JC et al. Microrheology probes length scale dependent rheology. Phys Rev Lett. 2006;96(11):118104.

[51] Brangwynne CP, MacKintosh FC, Kumar S, Geisse NA, Talbot J, Mahadevan L et al. Microtubules can bear enhanced compressive loads in living cells because of lateral reinforcement. J Cell Biol.2006;173(5):733-741. 